\title{
Complications after biopsy of renal transplant - the role of ultrasound examination
}

Authors: Olga Pustelniak ${ }^{1}$, Katarzyna Drelich ${ }^{2}$, Maryla Kuczyńska ${ }^{3}$ (mentor)

1 Students' Scientific Society at the Department of Interventional Radiology and Neuroradiology, Medical University of Lublin, Lublin, Poland

${ }^{2}$ Students' Scientific Society at the Department of Pediatric Radiology, Medical University of Lublin, Lublin, Poland

${ }^{3}$ Department of Interventional Radiology and Neuroradiology, Medical University of Lublin, Lublin, Poland

DOI: https://doi.org/10.26800/LV-142-supp5-34

\section{Introduction:}

Kidney transplantation is the most effective method of renal replacement therapy - the transplanted organ is able to take over all the functions performed by a healthy kidney. The function of the kidney after transplantation should be regularly observed in laboratory tests and imaging examinations. However, in some patients, it is necessary to perform a posttransplant biopsy in order to clearly identify the pathology that may lead to damage to the function of the kidney or transplant rejection.

Aim:

The aim of the study was to assess the type of complications occurring after a biopsy of a transplanted kidney and to assess the effectiveness of ultrasound in diagnosing them.

\section{Materials \& Methods:}

A retrospective evaluation of the results of US examinations in patients after biopsy of a transplanted kidney was performed in terms of the incidence of complications. All ultrasound examinations were performed in the radiology department with the use of Logiq 7 and Logiq 9 devices in B-mode presentation and in color and spectral Doppler options.

\section{Results:}

Complications after biopsy were found in 9 patients: 6 arteriovenous fistulas and 3 hematomas. One of the patients required the embolization of the damaged vessel by the endovascular method.

\section{Conclusion:}

Ultrasound examination with the use of Doppler option is an effective method to detect complications after a biopsy of a transplanted kidney, which allows for qualification for endovascular treatment.

Keywords: ultrasound, renal biopsy, transplantation 